\title{
Innovative Artificial Neural Networks-Based Decision Support System for Heart Diseases Diagnosis
}

\author{
Sameh Ghwanmeh, Adel Mohammad, Ali Al-Ibrahim \\ Computer Science Department, Faculty of Information Technology, WISE University, Amman, Jordan. \\ Email:sameh@wise.edu.jo
}

Received March 12 $2^{\text {th }}, 2012$; revised May $13^{\text {th }}, 2013$; accepted May $29^{\text {th }}, 2013$

Copyright (C) 2013 Sameh Ghwanmeh et al. This is an open access article distributed under the Creative Commons Attribution License, which permits unrestricted use, distribution, and reproduction in any medium, provided the original work is properly cited.

\begin{abstract}
Heart diagnosis is not always possible at every medical center, especially in the rural areas where less support and care, due to lack of advanced heart diagnosis equipment. Also, physician intuition and experience are not always sufficient to achieve high quality medical procedures results. Therefore, medical errors and undesirable results are reasons for a need for unconventional computer-based diagnosis systems, which in turns reduce medical fatal errors, increasing the patient safety and save lives. The proposed solution, which is based on an Artificial Neural Networks (ANNs), provides a decision support system to identify three main heart diseases: mitral stenosis, aortic stenosis and ventricular septal defect. Furthermore, the system deals with an encouraging opportunity to develop an operational screening and testing device for heart disease diagnosis and can deliver great assistance for clinicians to make advanced heart diagnosis. Using real medical data, series of experiments have been conducted to examine the performance and accuracy of the proposed solution. Compared results revealed that the system performance and accuracy are acceptable, with a heart diseases classification accuracy of $92 \%$.
\end{abstract}

Keywords: Heart Disease Diagnosis; Classification Accuracy; ANNs; Decision Support System; Knowledge Base

\section{Introduction}

Human heart can be described as a compound body organ contains muscles together with biological nerves. Human heart pumps nearly 51 of blood in the body providing the human body with renewed materials [1]. Medical research literature shows that there is much interest from the scientific researchers in implementing the human intelligence, including ANNs, in medical devices. The human heart operation is composite and any failure is risky to human lives. Hence, heart diagnose systems have been a main concern to the scientific researchers in the last decades [2]. It is not possible to use linear systems to perform the heart diagnosis. Neural Networks are a non-linear behavior structure and have been proven to be effective in the diagnosis systems [3].

Advanced heart diagnosis equipment is not always available in every medical center, especially in the rural areas where less support and care. Moreover, it is not possible for many people to travel to regional medical centers where high quality hospital services are affordable. Physician intuition and experience are not always enough to attain high quality medical results. Conse- quently, medical errors and undesirable results are reasons for a need for a state-of-the-art computer-based diagnosis systems, which in turns reduce medical fatal errors, increase patient safety and save lives [4,5]. Additionally, intuition and experience are frequently used by physicians to reach the medical verdicts instead of knowledge base. Consequently, unsolicited preconceptions and excessive medical cost are produced which may portend the patients' lives [6,7].

In this paper, it is proposed to build an ANN-based decision support system for heart diseases diagnosis. The system comprises two main components: a software part and a hardware part, which consists of a simple electronic stethoscope with a matching impedance electronic circuit. It is expected that the proposed system would benefit medical physicians to diagnose heart sound signals and checking the up-normality. Also, it is anticipated that the proposed system would provide innovative diagnostic tool to classify the heart diseases: mitral stenosis (MS), aortic stenosis (AS) and ventricular septal defect (ASD). Also, the system would offer a promising opportunity to develop an operational screening and testing device for heart disease diagnosis. The perform- 
ance, classification accuracy and effectiveness of the proposed NN-based decision support system have been examined. Series of experiments were carried out using real medical data from the Royal Medical Services. The testing experiments have been conducted to provide a clear comparison between the proposed system and other systems and techniques. Performance evaluation process has been conducted to reveal the best value of heart diseases classification accuracy.

\section{Literature Survey}

Generally, ANNs have been used in many areas of medicine, such as cardiology [8], Electroencephalography [9], Pulmonology [10], Genetics [11], Clinical chemistry, Pathology, Ophthalmology, Obstetrics and Gynecology [12]. Further, considerable research has been conducted on heart diagnosis systems $[13,14]$. However, most of these studies deal with only one specific disease area, dedicated to valvular diseases. The following provides an overview of related studies:

- Y. Hongmei, et al. present "A multilayer perceptron-based medical decision support system for heart disease diagnosis" study. The study deals with identifying 5 heart diseases based on heart auscultation: hypertension, coronary heart disease, rheumatic valvular heart disease, chronic cor pulmonale, and congenital heart disease $[15,16]$.

- S. Soumya, presents "System for Diagnosing Valvular Heart Disease using Heart Sounds" study. The study deals with a diagnosis scheme based on heart auscultation for aortic regurgitation, aortic stenosis, mitral regurgitation and mitral stenosis $[17,18]$.

- M. Zamri, et al. present "Wavelet Analysis and Classification of Mitral Regurgitation and Normal Heart Sounds" study. The study deals with mitral regurgitation diagnose based on ANNs [19].

- I. Turkoglu and A. Arslan, present "An Intelligent Pattern Recognition System Based on Neural Network and Wavelet Decomposition for interpretation of heart sounds" study. The study deals with aortic stenosis, mitral regurgitation, atrial septal defect mitral stenosis and aortic regurgitation disease diagnosis [20].

- Other related studies can be seen in [21-23].

\section{Methods and Materials}

\subsection{Artificial Neural Network}

Due to high assets of the human brain NN, scientists try to relate the principal of synaptic approaches to computer science and its applications. Thus, they the principal of ANN is introduced. The elementary computational element (model neuron) is often called a node or unit (Figure 1). It accepts input from other or from an external

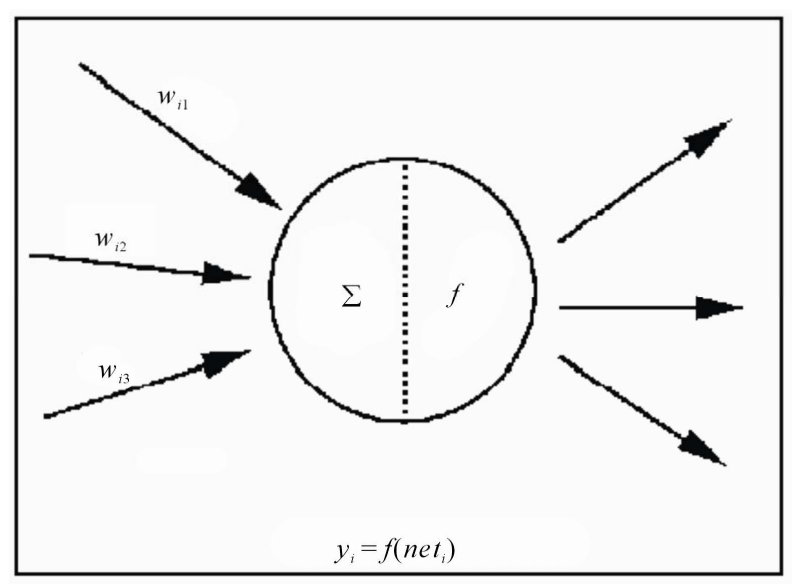

Figure 1. ANN basic building layout.

source. Each input has an associated weight w, which can be reformed, so as to model synaptic education [23]. The unit computes some function $f$ of the weighted sum of its inputs (Equation (1)):

$$
Y_{i}=f\left(\Sigma_{j} w_{i j} y_{j}\right)
$$

where

- The weighted sum $\Sigma_{j} w_{i j} y_{j}$ is called the net input to unit $i$, often written net $t_{i}$.

- Note that $w_{i j}$ refers to the weight from unit $j$ to unit $i$ (not the other way around).

- The function $f$ is the unit's activation function. In the simplest case, $f$ is the identity function, and the unit's output is just its net input. This is called a linear unit [23].

\subsection{Back Propagation}

Back-propagation was formed by specifying the Widrow-Hoff learning rule to multiple-layer networks and nonlinear differentiable transfer functions. Input vectors and the corresponding target vectors are used to train a network until it can be able to rough a function, associate input vectors with specific output vectors, or classify input vectors in an appropriate way as defined by the user. Networks with biases, a sigmoid layer, and a linear output layer are capable of approximating any function with a finite number of discontinuities. Appropriately, trained back propagation networks have a tendency to provide reasonable answers when presented with inputs that they have never seen. Naturally, a new input leads to an output similar to the correct output for input vectors used in training that are similar to the new input being presented. This generalization property makes it possible to train a network on a representative set of input/target pairs and get upright results without training the network on all possible input/output pairs. There are two features of the Neural Network Matlab Toolbox that are designed to improve network generalization: regularization and 
early stopping [23].

\subsection{NN Applications in Medicine}

$\mathrm{NN}$ have been used progressively in the field of medicine. They are predominantly well suitable to problems with a high degree of difficulty for which there is no algorithmic solution or the solution is too complex for traditional techniques to be determined. Successful implementation of this technology in medical areas can be seen in drug development, patient diagnosis, and image analysis. Two major keystones are: the detection of coronary artery disease and the processing of Electroencephalography signals. The following are common application in which NNs are used: cardiology, analysis of electroencephalography, pulmonology, genetics, clinical chemistry, Pathology, ophthalmology and obstetrics, gynecology.

\subsection{Proposed System Structure}

The proposed ANN-based decision support system is composed of two parts: hardware and software. The hardware is constructed to represent the pattern recognition sensor to collect heart sounds. It comprises of stethoscope, microphone and sound cable (Figure 2). Since the collected sound by stethoscope is low amplitude level, a microphone capsule is inserted between the stethoscope and the PCI sound card interface to amplify the sound waves fed by the stethoscope. The GUI software part is implemented using Matlab and ANN toolbox. The software has been built by Matlab. Matlab includes different Toolboxes. Neural networks toolbox is used to build the presented intelligent information system to diagnose heart diseases.

\subsubsection{Data Assembly}

The testing data have been collected based on two different sources. The first source is provided by the Jordanian Royal medical services. While the second source is the recorded samples directly provided from the patient tested on the proposed system.

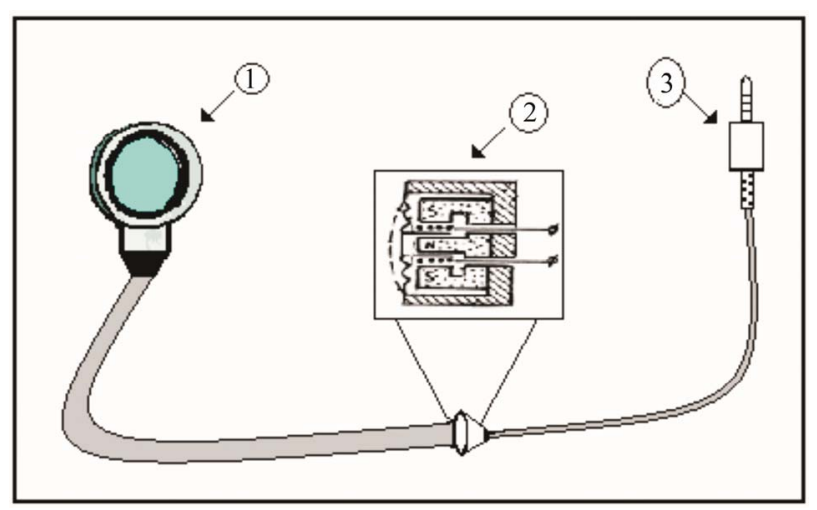

Figure 2. The Hardware design of the system.

\subsubsection{Extracting the Signal Features}

The Matlab Fast Fourier transform (FFT) is a mathematical technique used for transforming a function in time domain into a function of frequency domain. FFT is useful for analysis of time-dependent phenomena. Therefore, it is important to assess the frequency distribution of the power in heart sound signal in alike way to the human ear which workouts that capacity in the hearing process. Accordingly, useful information is booked by separating the first part of the spectrum. FFT is used to extract the main sound features. FFT assumptions employed in the proposed system are shown below. The Matlab procedure which has been employed in this process is shown in Figure 3. Two different sources of the heart signals are used: a collection of a recorded set of sound signals and stethoscope signals. Each recoded analog signals is passed to the FFT to produce the heart signal in frequency domain (Figure 4-7). Similarly, the signal produced from the stethoscope is passed to the FFT to produce the heart signal in frequency domain (Figures 8 and $\mathbf{9}$ ).

- The sample frequency was set to $8000 \mathrm{~Hz}$.

- Perform FFT on the wave file $(100,000$ samples are taken).

- Taking the absolute value of the important (beginning 5000) part of data in the new matrix.

- Split the FFT matrix into 50 bins and get the average of each.

- Standardize the return value by dividing the matrix by its sum.

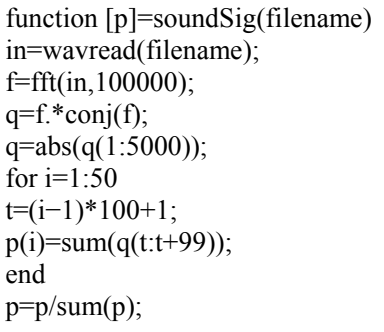

Figure 3. The Matlab procedure employed to extract the FFT features.

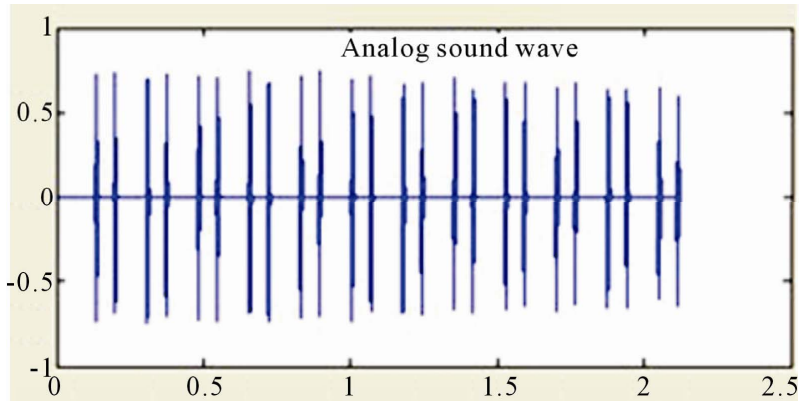

Figure 4. Analog signal for a normal heart (recorded sample). 


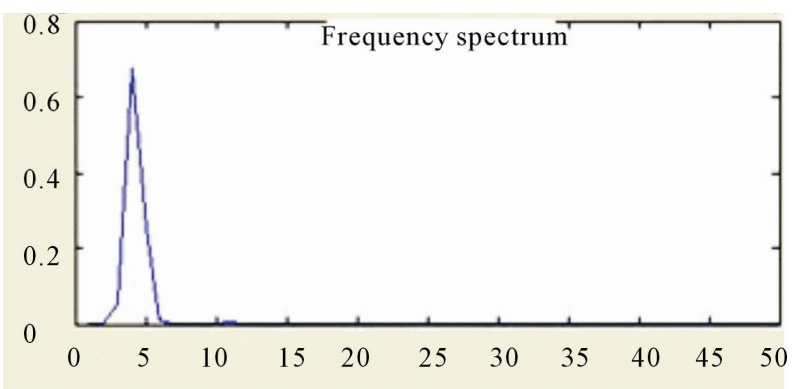

Figure 5. FFT signal for a normal heart (recorded sample).

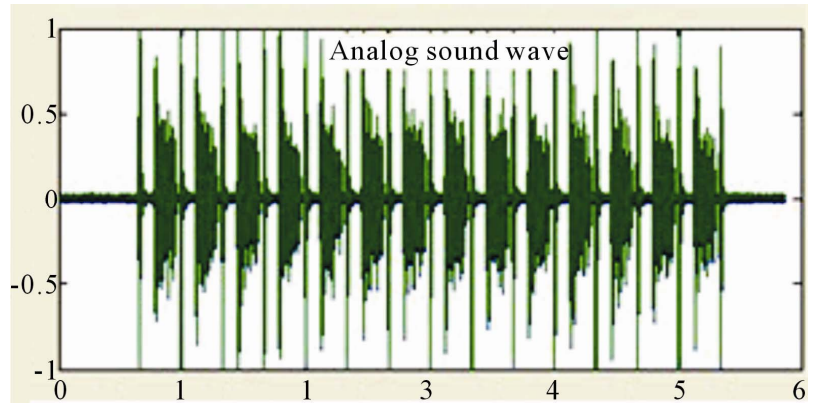

Figure 6. Analog signal for an up normal heart (recorded sample).

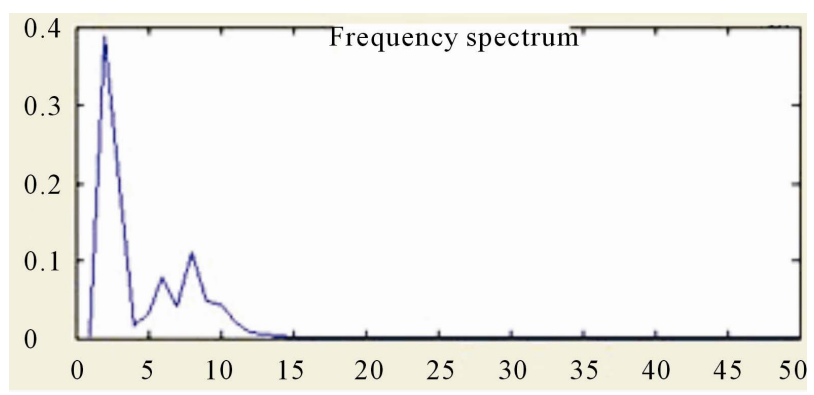

Figure 7. FFT signal for an up normal heart (recorded sample).

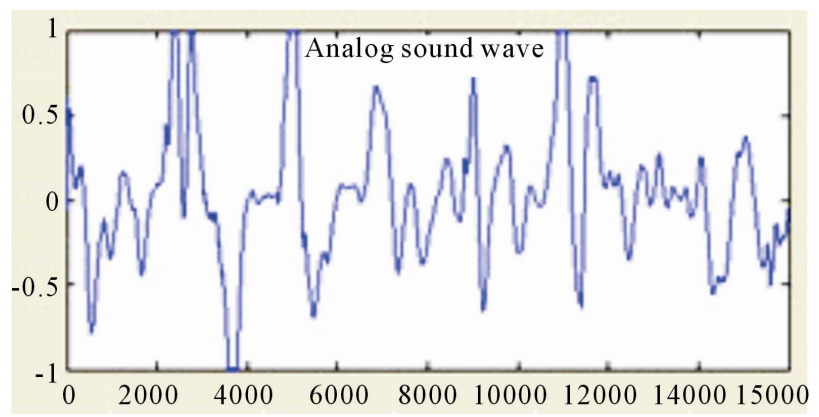

Figure 8. Analog signal for a normal heart (stethoscope sample).

\subsubsection{ANN Training}

Three ANNs are built: mitral stenosis disease ANN, ventricular septal defect disease ANN and aortic stenosis disease ANN. The ANNs parameters are initialized based on default values, including: epochs (number of itera-

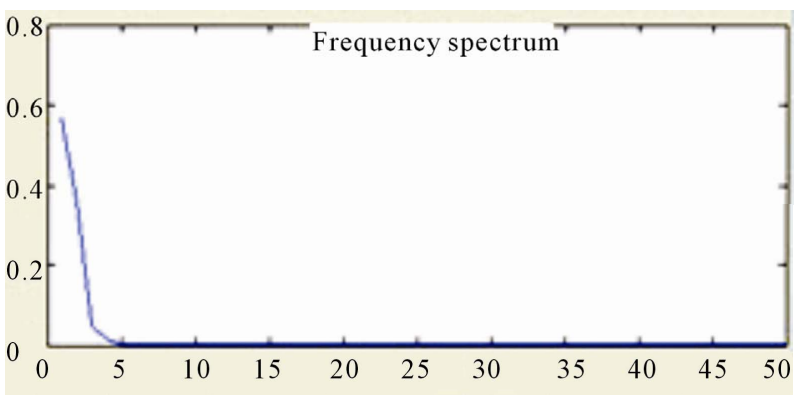

Figure 9. FFT signal for a normal heart (stethoscope sample).

tions), goal (performance function) and ANN show number.

\section{Experimental Results and Discussion}

Series of experiments with different samples have been conducted to measure the effectiveness and accurateness of the proposed system. To ensure the correctness of the knowledge base and outputs, ANN are trained repeatedly and its parameters are modified where is needed. Number of trained samples, with wide range of variations and noise effect in each class, are also increased to enhance the system accuracy. Results show that accurate outputs, with $98 \%$ classification accuracy, are resulted with the trained samples, while, the system produces acceptable results for non-trained samples, with $92 \%$ classification accuracy. Additional experiments are carried out using external stethoscope and the output is accurate. However, it can be seen (Figure 10) that the shape of the recorded sound waveform looks little different compared with the stored one. The reason behind this is that the samples waveforms were sampled at different rate. The system output for normal heart using stethoscope is shown in Figure 10.

Overall, the results revealed that all heart diseases of MS, AS and VSD are successfully and accurately identified with the proposed system, as shown in (Figures 10-13). Compared with previous studies presented in [21-24], which are mainly focused on valvular heart diseases, the proposed system delivers higher classification accuracy for both valvular and non-valvular heart diseases.

\section{Comparison Study}

Hongmei Yana et al. (Table 1) present a research study entitled "A multilayer perceptron-based medical decision support system for heart disease diagnosis" which deals with diagnosing of five heart diseases based on heart auscultation: hypertension, coronary heart disease, rheumatic valvular heart disease, chronic cor pulmonale, and congenital heart disease. Three different evaluation methods were adopted to assess the performance of the 


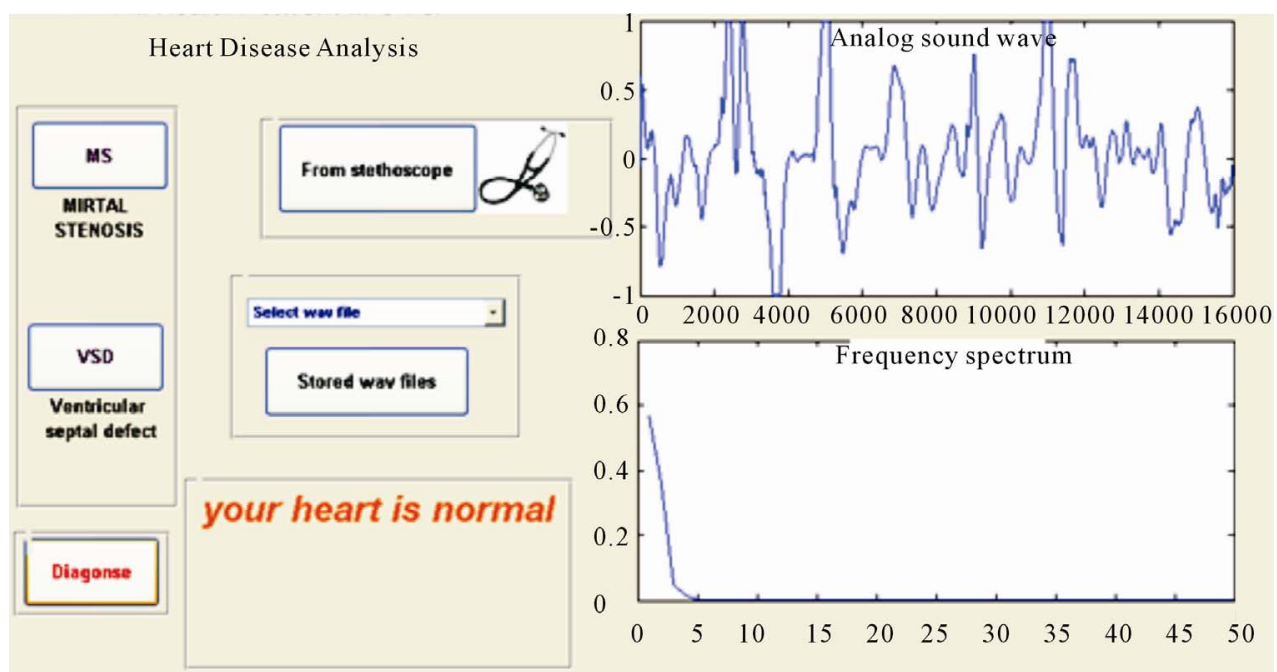

Figure 10. System output for normal heart using stethoscope.

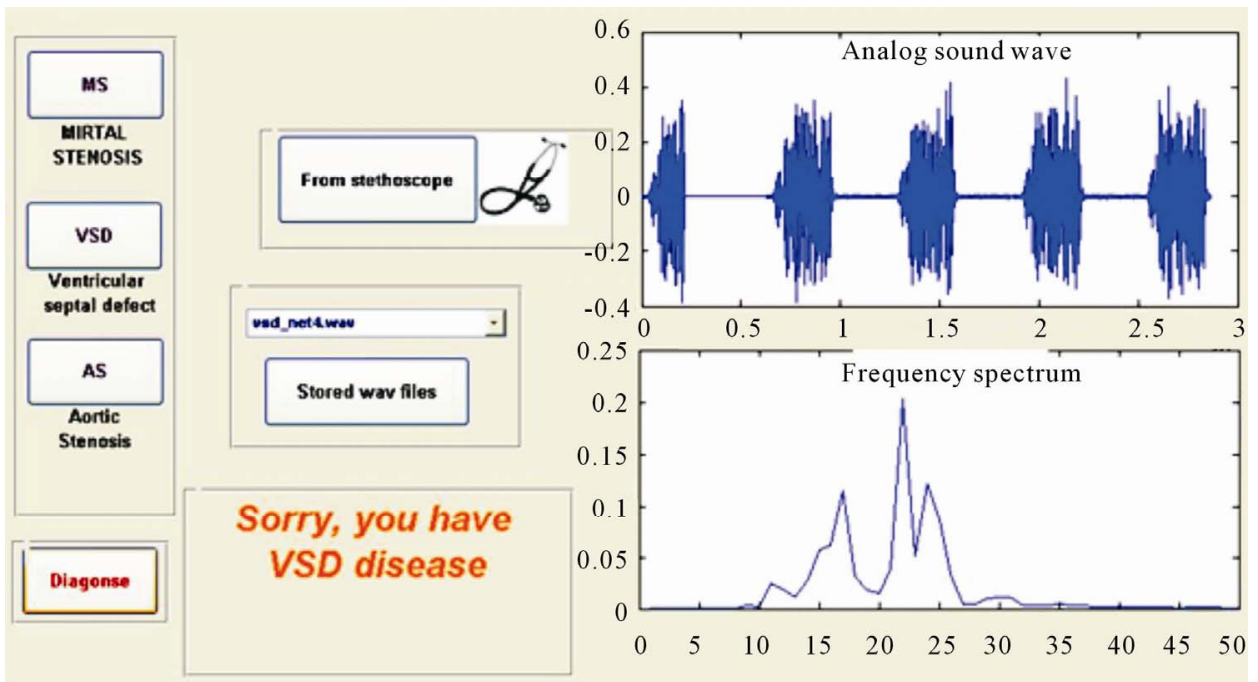

Figure 11. System output for “ventricular septal defect” heart disease.

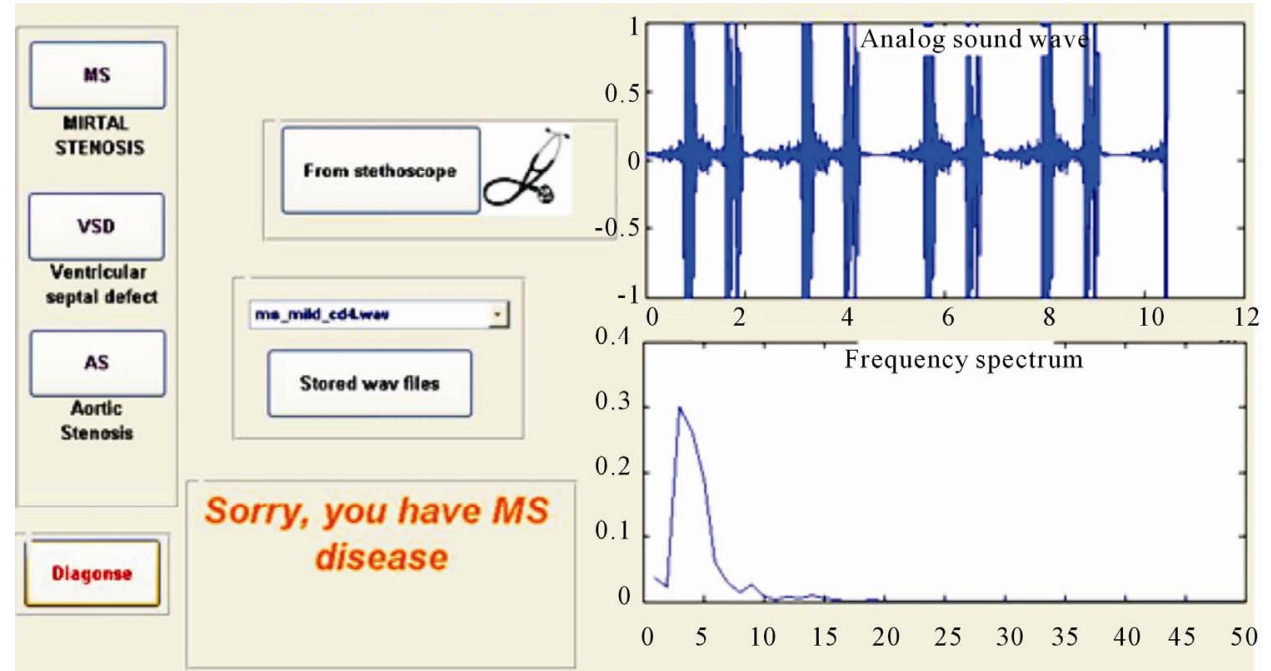

Figure 12. System output for "mitral stenosis" heart disease. 


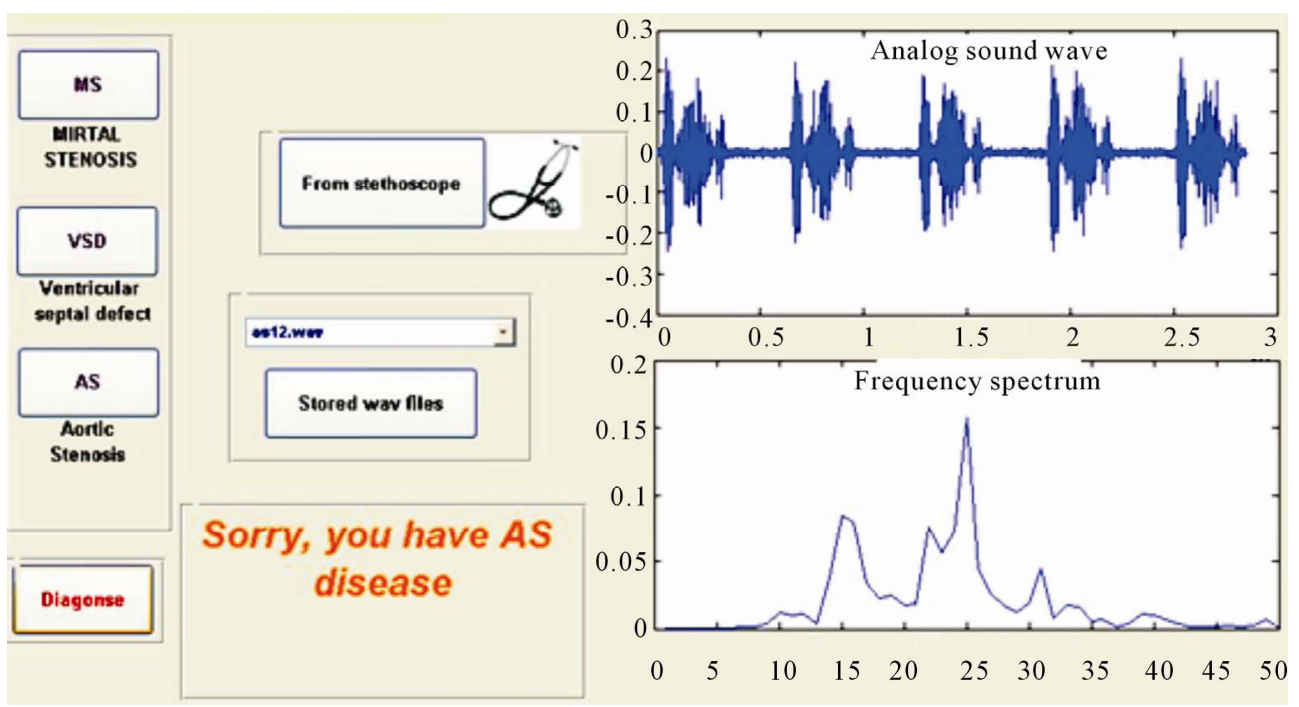

Figure 13. System output for “aortic stenosis” heart disease.

Table 1. Detailed comparison study results.

\begin{tabular}{cccc}
\hline Reference & Type of heart diseases & $\begin{array}{c}\text { \# of heart } \\
\text { diseases }\end{array}$ & $\begin{array}{c}\% \\
\text { accuracy }\end{array}$ \\
\hline$[16]$ & Valvular diseases & 5 & 90 \\
{$[18]$} & Valvular diseases & 4 & 81 \\
{$[19]$} & Valvular diseases & 1 & 94.2 \\
{$[20]$} & Valvular diseases & 5 & 95.5 \\
Proposed system & $\begin{array}{c}\text { Valvular diseases and non } \\
\text { valvular diseases }\end{array}$ & 3 & 92 \\
\hline
\end{tabular}

proposed decision support system. Results revealed that that the system has accuracy for all five heart diseases of about $(90 \%)$.

Soumya Sakha Tripathy (Table 1) presents a thesis entitled "System for Diagnosing Valvular Heart Disease using Heart Sounds". The thesis depicts a diagnosis system based on heart auscultation for Aortic Regurgitation, Aortic stenosis, Mitral regurgitation and Mitral stenosis. The classification was carried out by a feed-forward neural network with back-propagation learning algorithm. Results revealed that the classification accuracy is about $81 \%$.

Zamri, et al. (Table 1) present a research paper entitled "Wavelet Analysis and Classification of Mitral Regurgitation and Normal Heart Sounds". The paper explains the Mitral Regurgitation diagnose based on Artificial Neural Networks. The features from heart sounds were obtained from integral wavelet transform and used to train and test the artificial neural networks. The ANN was trained, with a classification accuracy of $94.2 \%$. The heart sound data was recorded using an electronic stethoscope. A multilayer perceptron neural network with an input, one hidden and an output layer were used to classify the heart sounds.
I. Turkoglu and A. Arsaln (Table 1) present a research paper entitled "An Intelligent Pattern Recognition System Based on Neural Network and Wavelet Decomposition for interpretation of heart sounds". The study deals with Aortic stenosis, Mitral regurgitation, atrial septal defect, Mitral stenosis and Aortic regurgitation disease diagnosis. Results show that the accuracy is averaged for all heart diseases with a value of $95.5 \%$.

From the above comparison study, it can be seen that the classification accuracy never reached $100 \%$ and it is in the range of $81 \%$ to $95.5 \%$. However, the proposed system has produced results with $92 \%$ accuracy. The compared studies dealt with different heart diseases and most of them are valvular diseases. Where, the proposed system deals with two valvular diseases (MS \& AS) and one non valvular disease (VSD). Some of the previous studies have used different feature extraction methods like Wavelet transform, while, in this study, FFT has been used. Additionally, the compared studies have used different neural network techniques, including multilayer perceptron ANNs. Whereas, in this study, the back propagation technique has been adopted (Table 1).

\section{Conclusion}

An innovative decision support system which is based on ANN has been implemented and tested to classify three main heart diseases: mitral stenosis, aortic stenosis and ventricular septal defect. Furthermore, the system offers a promising opportunity to develop an operational screening and testing device for heart disease diagnosis. Also, the system can deliver great assistance for clinicians to make advanced heart sound diagnosis. Using real medical data, series of experiments have been conducted, to examine the performance and accuracy of the proposed system. Compared results revealed that the system 
performance and accuracy are excellent, with a heart diseases classification accuracy of $92 \%$. Compared with previous studies, which are mainly focused on valvular heart diseases, results show that the proposed system delivers higher classification accuracy for both valvular and non-valvular heart diseases. Overall, the results presented show that all heart diseases of MS, AS and VSD are successfully and accurately identified with the proposed system. The proposed system can be used as great assistance to physicians in realization accurate and unfailing detecting heart problems at early stages and prevent patients going to proficient cardiologists.

More work is planned to enrich the ANN knowledge base with more heart disease samples and enlarge the system to cover a wide range of heart diseases with high accuracy. Additional NN training techniques can be adopted, including linear vector quantization (LVQ), perception and dynamic NN. Further, it is proposed to embed the system components in one commercial product.

\section{Acknowledgements}

The authors gratefully acknowledge and highly appreciate the financial support and the remarkable resources provided by WISE University, Amman, Jordan.

\section{REFERENCES}

[1] S. Abdul, V. D. Bhagile, R. R. Manza and R. Ramteke, "Diagnosis and Medical Prescription of Heart Disease Using Support Vector Machine and Feedforward Backpropagation Technique," International Journal on Computer Science \& Engineering, Vol. 1, No. 6, 2010, pp. 2150-2159.

[2] H. Uğuz, "A Biomedical System Based on Artificial Neural Network and Principal Component Analysis for Diagnosis of the Heart Valve Diseases," Journal of Medical Systems, Vol. 36, No. 1, 2012, pp. 61-72. doi:10.1007/s10916-010-9446-7

[3] M. Lomsky, P. Gjertsson, L. Johansson, J. Richter, M. Ohlsson, D. Tout, A. Aswegen, S. Underwood and L. Edenbrandt, "Evaluation of a Decision Support System for Interpretation of Myocardial Perfusion Gated SPECT," European Journal of Nuclear Medicine \& Molecular Imaging, Vol. 35, No. 8, 2008, pp. 1523-1529. doi:10.1007/s00259-008-0746-9

[4] A. V. S. Kumar, "Diagnosis of Heart Diseases Using Fuzzy Resolution Mechanism," Journal of Artificial Intelligence, Vol. 5, No. 1, 2012, pp. 47-55. doi:10.3923/jai.2012.47.55

[5] Q. K. Al-Shayea, "Artificial Neural Networks in Medical Diagnosis," International Journal of Computer Science Issues, Vol. 8, No. 2, 2011, pp. 150-154.

[6] N. Santhiyakumari, P. Rajendran and M. Madheswaran, "Medical Decision-Making System of Ultrasound Carotid
Artery Intima-Media Thickness Using Neural Networks," Journal of Digital Imaging, Vol. 24, No. 6, 2011, pp. 1112 1125. doi:10.1007/s10278-010-9356-8

[7] Z. L. Liao, B. Wang, X. W. Xia and P. M. Hannam, "Environmental Emergency Decision Support System Based on Artificial Neural Network," Safety Science, Vol. 50, No. 1, 2012, pp. 150-163. doi:10.1016/j.ssci.2011.07.014

[8] http://en.wikipedia.org/wiki/Data_classification

[9] http://www.doc.ic.ac.uk/ nd/surprise_96/journal/vol4/cs1 1/report.html\#

[10] http://en.wikipedia.org/wiki/Heart_sound\#S1

[11] http://www.cvphysiology.com/Arrhythmias/A008.htm

[12] P. Kornel, M. Bela, S. Rainer, D. Zalan, T. Zsolt and F. Janos, "Application of Neural Networks in Medicine N a Rview". http://www.medscimonit.com/pub/vol_4/no_3/694.pdf

[13] R. Das, I. Turkoglu and A. Sengur, "Diagnosis of Valvular Heart Disease through Neural Networks Ensembles," Computer Methods \& Programs in Biomedicine, Vol. 93, No. 2, 2009, pp. 185-191. doi:10.1016/j.cmpb.2008.09.005

[14] S. Mehrabi, M. Maghsoudloo, H. Arabalibeik, R. Noormand and Y. Nozari, "Application of Multilayer Perceptron and Radial Basis Function Neural Networks in Differentiating between Chronic Obstructive Pulmonary and Congestive Heart Failure Diseases," Expert Systems with Applications, Vol. 36, No. 3, 2009, pp. 6956-6959. doi:10.1016/j.eswa.2008.08.039

[15] http://www.calsci.com/heartattacks.html

[16] H. Yana, Y. T. Jiangb, J. Zhenge, C. Pengc and Q. Lid. "A Multilayer Perceptron-Based Medical Decision Support System for Heart Disease Diagnosis," Elsevier Expert Systems with Applications, Vol. 30, No. 2, 2006, pp. 272-281. doi:10.1016/j.eswa.2005.07.022

[17] http://www.bsignetics.com

[18] S. T. Soumya, "System for Diagnosing Valvular Heart Disease Using Heart Sounds," 2005.

[19] Z. M. Zin, et al. "Wavelet Analysis and Classification of Mitral Regurgitation and Normal Heart Sounds Based on Artificial Neural Networks," Proceedings of the 7th International Symposium on Signal Processing and Its Applications, 1-4 July 2003, pp. 619-620. doi:10.1109/ISSPA.2003.1224955

[20] I. Turkoglu and A. Arsaln, “An Intelligent Pattern Recognition System Based on Neural Network and Wavelet Decomposition for Interpretation of Heart Sounds," Proceedings of the 23rd Annual Conference of the IEEE, Turkey, 25-28 October 2001, pp. 1747-1750.

[21] I. Turkoglu, A. Arslan and E. Ilkay, “A Wavelet Neural Network for the Detection of Heart Valve Diseases," Expert Systems, Vol. 20, No. 1, 2003, pp. 1-7. doi:10.1111/1468-0394.00219

[22] K. S. Kavitha, K. V. Ramakrishnan and M. K. Singh, "Modeling and Design of Evolutionary Neural Network for Heart Disease Detection," International Journal of Computer Science Issues, Vol. 7, No. 5, 2010, pp. 272283. 
[23] R. Das, I. Turkoglu and A. Sengur, "Diagnosis of Valvular Heart Disease through Neural Networks Ensembles," Computer Methods and Programs in Biomedicine, Vol.
93, No. 2, 2009, pp. 185-191.

doi:10.1016/j.cmpb.2008.09.005 